\title{
Novel melanoma therapy
}

\author{
Eddy C. Hsueh* and Kalyan C. Gorantla
}

\begin{abstract}
With the rapid succession of new effective agents for melanoma in the recent years, the paradigm for treatment of metastatic melanoma is changing. The success of combining multiple effective agents compared with outcomes of monotherapy also brings increasing complexity in the treatment algorithm for various subsets of metastatic melanoma patients. We reviewed the recent reports on novel melanoma therapy to shed light on rational decision-making in treating these patients.
\end{abstract}

Keywords: Metastatic melanoma, Checkpoint inhibitors, Targeted therapy

\section{Background}

Recent rapid succession of breakthroughs in melanoma therapy has dramatically changed the treatment paradigm for metastatic melanoma over the last 5 years. The remarkable success of immune checkpoint inhibitors in metastatic melanoma treatment has validated immunotherapy as a major modality for cancer treatment in addition to surgery, chemotherapy, and radiation therapy. The elucidation of molecular pathways in cancer biology has given rise to targeted therapy with dramatic responses in select metastatic melanoma patients. The development of oncolytic virus in melanoma treatment has brought forth the first FDA-approved oncolytic viral therapy for cancer. With these exciting breakthroughs in melanoma therapy and continuing to emerge novel therapies for melanoma, the landscape of therapeutic options for patients with advanced stages of melanoma has drastically changed in comparison to options available for 30 plus years until 2011. In 2011, the FDA approved ipilimumab, a cytotoxic T-lymphocyte 4 (CTLA-4) inhibitor, based on the results of a 3-arm randomized phase III trial comparing a gp100 vaccine alone, ipilimumab alone, and the combination of vaccine with ipilimumab [1]. Subsequently, vemurafenib, a BRAF inhibitor, was approved in the same year for patients with metastatic melanoma harboring BRAF mutation [2, 3]. In 2013, the combination of BRAF inhibitor, dabrafenib, and MEKinhibitor, trametinib, was

\footnotetext{
*Correspondence: hsuehec@slu.edu

Division of General Surgery, Department of Surgery, Saint Louis University, 3635 Vista at Grand Blvd, St. Louis, MO 63110, USA
}

approved for BRAF mutation positive metastatic melanoma patients [4]. Approval of two inhibitors of the programmed death 1 (PD-1) receptor, pembrolizumab and nivolumab, were granted in $2014[5,6]$. This review will attempt to summarize the advancement in novel melanoma therapy in the literature from the recent years and provide a reference for treatment decision consideration.

\section{Targeted therapy}

Mutation in the serine-threonine kinase BRAF was observed in close to $50 \%$ of metastatic melanoma lesions, mostly of the valine to glutamine substitution in codon 600 (V600E) [7]. Other less common BRAF mutations with lysine (V600K) or arginine (V600R) substitutions have also been reported. Vemurafenib and dabrafenib are the currently FDA-approved BRAF inhibitors that target these mutations for the treatment of melanoma. BRAF inhibitors have shown high rates of response (48-59\%) in phase II and III trials but with limited duration [2, 3, 8]. The median progression-free survival (PFS) ranges from 5.1 to 6.8 months $[8,9]$. The lack of durability of response is due to the development of resistance, mainly reactivation of the mitogen-activated protein kinase (MAPK) pathway. Although reactivation of MAPK pathway was observed in over two-thirds of BRAF-inhibitor progressing tumors, amplification of parallel signaling networks such as the PI3K-PTEN-AKT pathway were also found [10-12]. Based on preclinical models, combined treatment with BRAF and MEK inhibitors might attenuate development of resistance by blocking reactivation of the MAPK pathway induced by single-agent 
BRAF inhibitors $[13,14]$. As a first-line monotherapy, treatment with MEK inhibitor trametinib has also shown similar response rate (48 \%) and PFS (4.8 months) compared with BRAF inhibitors [15].

Combination of BRAF/MEK inhibition with dabrafenib (BRAF inhibitor) and trametinib (MEK inhibitor) was evaluated in a phase I/II study [4]. The absence of drug-drug interaction was confirmed in 8 patients with repeated doses of trametinib and a single dose of dabrafenib. Escalating doses of dabrafenib (75 and $150 \mathrm{mg}$ twice daily [BID]) in combination with trametinib $(1,1.5$ and $2 \mathrm{mg}$ every day [QD]) were evaluated in 77 patients to determine toxicity profile and pharmacokinetic activity. In the phase II expansion study, 162 advanced melanoma patients with BRAFV600 mutation and no prior BRAF targeted therapy were assigned 1:1:1 to receive dabrafenib (150 mg QD) and trametinib (either 1 or $2 \mathrm{mg}$ QD) or dabrafenib (150 mg QD) monotherapy. Median PFS for those in the combination $150 / 2$ group was 9.4 versus 5.8 months for patients who received dabrafenib monotherapy (hazard ratio $[\mathrm{HR}]$ for progression or death, 0.39; $\mathrm{p}<0.001)$. In a subsequent phase III study (COMBI-d), 423 previously untreated and unresectable stage IIIC or stage IV melanoma patients with BRAF V600E or V600K mutation were randomized to receive combination of dabrafenib (150 mg BID) and trametinib (2 mg QD) or dabrafenib and placebo [16]. With a median follow-up of 9 months, median PFS was 9.3 months in combination group and 8.8 months in dabrafenib-only group (HR 0.75; 95 \% Confidence Interval [CI] 0.570.99; $\mathrm{p}=0.03$ ). Similar rates of adverse events (AEs) were observed between the two groups. However, the rate of cutaneous squamous-cell carcinoma was lower in the combination group compared with dabrafenibonly group ( 2 versus $9 \%$ ). Pyrexia were more common (51 versus $28 \%$ ) and with more severity (grade 3, 6 versus $2 \%$ ) in the combination group. In a follow-up report, the median overall survival (OS) was 25.1 months in the combination group versus 18.7 months in the dabrafenib only group (HR $0.71,95 \%$ CI $0.55-0.92 ; \mathrm{p}=0.0107$ ) [17]. The median PFS was 11.0 months in the combination group and 8.8 months in the dabrafenib only group (HR $0.67,95 \%$ CI 0.53-0.84; $\mathrm{p}=0.0004)$. In a second phase III trial, combination of dabrafenib (150 mg BID) and trametinib (2 mg QD) were compared with vemurafenib (960 mg BID) orally as first-line therapy (COMBI-v) [18]. The combination also improved OS, PFS, and overall response rates (ORR) compared with vemurafenib alone group. The OS rate at 12 months was $72 \%$ in the combination group and $65 \%$ in the vemurafenib group (HR $0.69 ; 95 \%$ CI $0.53-0.89 ; \mathrm{p}=0.005)$. Median PFS was 11.4 months in the combination group and 7.3 months in the vemurafenib group (HR 0.56; $95 \% \mathrm{CI}, 0.46-0.69$; $\mathrm{p}<0.001)$. ORR rate was $64 \%$ in the combination group and $51 \%$ in the vemurafenib group $(\mathrm{p}<0.001)$. Cutaneous squamous-cell carcinoma and keratoacanthoma occurred in $1 \%$ of patients in the combination group and $18 \%$ of those in the vemurafenib group. In all three studies, rates of AEs were similar between the combination group and the monotherapy group. However, AEs related to reactivation of the MAPK pathway were reduced in the combination group compared with BRAF inhibitor monotherapy group. Pyrexia was more common and more severe in the combination group.

Combination therapy with BRAF inhibitor vemurafenib and MEK inhibitor cobimetinib were also evaluated in clinical trials with similar ORR, PFS and OS as dabrafenib and trametinib combination. In the phase Ib BRIM7 trial, patients who progressed on vemurafenib $(\mathrm{n}=66)$ or never received BRAF inhibitor $(\mathrm{n}=63)$ were enrolled and treated on ten dosing regimens with vemurafenib 720 or $960 \mathrm{mg}$ BID continuously and cobimetinib 60, 80, or $100 \mathrm{mg}$ QD for either 14 days on and 14 days off, 21 days on and 7 days off, or continuously [19]. Dose-limiting toxic effects were observed in four patients: grade 3 fatigue for more than 7 days, grade 3 prolongation of QTc, grade 3 stomatitis and fatigue, and arthralgia and myalgia. The maximum tolerated dose was established as vemurafenib $960 \mathrm{mg}$ BID in combination with cobimetinib $60 \mathrm{mg}$ QD 21 days on and 7 days off. Confirmed objective responses were observed in 10 of 66 patients $(15 \%)$ who had recently progressed on vemurafenib, with a median PFS of 2.8 months. Confirmed objective responses were noted in 55 of $63 \mathrm{BRAF}$ inhibitor naive patients ( $87 \%$ ) with median PFS of 13.7 months. All 63 patients who had never received a BRAF inhibitor had evidence of target lesion reduction after combination treatment. Recent update to the study was reported with additional 11 months of follow up [20]. Median OS of 28.5 months in the vemurafenib-naive and 8.4 months in the vemurafenib-progressing patients was reported, with 2-year OS rates of 61.1 and $15.1 \%$ respectively. In the subsequent phase III coBRIM trial, 495 patients with treatment-naïve BRAF V600 mutation-positive melanoma were randomized to receive vemurafenib $(960 \mathrm{mg}$ BID) and cobimetinib (60 mg QD 21 days on and 7 days off) (combination group) or vemurafenib and placebo (control group) [21]. Median PFS was 9.9 months in the combination group and 6.2 months in the control group (HR 0.51; 95 \% CI 0.39-0.68; p < 0.001). ORR was $68 \%$ in the combination group as compared with $45 \%$ in the control group $(\mathrm{p}<0.001)$, with $10 \%$ complete response (CR) in the combination group and $4 \% \mathrm{CR}$ in the control group. The combination treatment was associated with higher incidence of grade 3 or higher AEs, as compared with control (65 versus $59 \%$ ). The incidence of secondary 
cutaneous squamous cell cancer was also decreased in the combination group compared with control group (2 versus $11 \%)$. Recent update with 8 months additional follow up reported a PFS of 12.3 months in the combination group compared with 7.3 months for the control group (HR 0.58) and ORR of 70 versus $50 \%$, respectively [22].

Another BRAF and MEK inhibitor combination in clinical trial is encorafenib and binimetinib. In a phase Ib/II trial patients with advanced BRAFV600 positive melanoma were treated with 400, 450 or $600 \mathrm{mg}$ QD of encorafenib and $45 \mathrm{mg}$ BID of binimetinib [23]. ORR of 74.5 \% with a median PFS of 11.3 months (95 \% CI 7.414.6) were observed in the BRAF inhibitor naïve patients $(\mathrm{n}=55)$. A 3-arm phase III trial (COLUMBUS) is ongoing where patients are randomized 1:1:1 to encorafenib $450 \mathrm{mg}$ QD and binimetinib $45 \mathrm{mg}$ BID, encorafenib alone at $300 \mathrm{mg}$ QD, and vemurafenib alone [ClinicalTrials.gov identifier: NCT01909453].

Recently, encouraging results were also reported with agent that targets NRAS mutant melanomas in an open label phase III trial randomizing NRAS-mutant melanoma patients with or without prior immunotherapy 2:1 to binimetinib $45 \mathrm{mg}$ BID $(\mathrm{n}=269)$ or DTIC $1000 \mathrm{mg} /$ $\mathrm{m}^{2}$ every 3 weeks $(\mathrm{n}=133)$ [24]. Confirmed ORR and disease control rate (DCR) were 15 and $58 \%$ for binimetinib group compared with 7 and $25 \%$ for DTIC group $(\mathrm{p}=0.015$ [ORR]; $\mathrm{p}<0.001[\mathrm{DCR}])$, respectively. Median duration of response on binimetinib was 6.9 months.

The studies reviewed above are briefly summarized in Table 1.

\section{Checkpoint inhibitors}

Pembrolizumab, the first anti-PD-1 antibody to be approved by the FDA, is a highly selective, humanized monoclonal IgG4-kappa isotype antibody against
PD-1. The results of the first phase I trial led to the FDA approval of pembrolizumab in ipilimumab-refractory metastatic melanoma patients in September 2014 [5, 25]. Patients with advanced melanoma with or without prior ipilimumab therapy $(\mathrm{n}=135)$ were enrolled (KEYNOTE-001) [25]. Doses of pembrolizumab at either 2 or $10 \mathrm{mg} / \mathrm{kg}$ given every 2 or 3 weeks were tested. ORR of $31-51$ and $81 \% 1$ year survival were observed. To address the utility of pembrolizumab in ipilimumab refractory patient population, an expansion cohort of 173 patients was enrolled in this phase I study [5]. Patients with advanced melanoma whose disease had progressed after $\geq 2$ ipilimumab doses were randomly assigned to $2 \mathrm{mg} /$ $\mathrm{kg}$ pembrolizumab every 3 weeks $(\mathrm{n}=89)$ or $10 \mathrm{mg} / \mathrm{kg}$ every 3 weeks $(n=84)$. ORR was $26 \%$ at both doses. The drug was well tolerated at both doses with similar safety profile and no drug-related deaths. Majority of patients in both doses had reduction of the target lesion size from baseline: $59(73 \%)$ patients in the pembrolizumab $2 \mathrm{mg} /$ $\mathrm{kg}$ group and $52(68 \%)$ in the $10 \mathrm{mg} / \mathrm{kg}$ group. The most common drug-related AEs of any grade in the $2 \mathrm{mg} / \mathrm{kg}$ and $10 \mathrm{mg} / \mathrm{kg}$ groups were fatigue (33 versus $37 \%$ ), pruritus (26 versus $19 \%$ ), and rash (18 versus $18 \%$ ). Further evaluation of pembrolizumab was conducted comparing with investigator-choice chemotherapy in ipilimumabrefractory patients (KEYNOTE-002) [26]. In a randomized phase 2 trial enrolling patients with progressive disease after $\geq 2$ ipilimumab doses 540 patients were randomly assigned (1:1:1) to receive pembrolizumab $2 \mathrm{mg} /$ $\mathrm{kg}$ or $10 \mathrm{mg} / \mathrm{kg}$ every 3 weeks or investigator-choice chemotherapy (paclitaxel plus carboplatin, paclitaxel, carboplatin, dacarbazine, or oral temozolomide). PFS was improved in pembrolizumab $2 \mathrm{mg} / \mathrm{kg}$ cohort (HR 0.57, $95 \%$ CI 0.45-0.73; p < 0.0001) and pembrolizumab $10 \mathrm{mg} / \mathrm{kg}$ cohort $(0.50,0.39-0.64 ; \mathrm{p}<0.0001)$ compared

Table 1 Recent targeted therapy study results in melanoma

\begin{tabular}{|c|c|c|c|c|c|}
\hline Study & Agent(s) & Phase of study & Median PFS (months) & OS & ORR (\%) \\
\hline \multirow[t]{2}{*}{ [4] } & Dabrafenib + trametinib & $\|$ & 9.4 & $79 \%$ at 1 year & 76 \\
\hline & Dabrafenib & & 5.8 & $70 \%$ at 1 year & 54 \\
\hline \multirow{2}{*}{$\begin{array}{l}{[16]} \\
{[17]}\end{array}$} & Dabrafenib + trametinib & III & 11.0 & 25.1 months & 67 \\
\hline & Dabrafenib & & 8.8 & 18.7 months & 51 \\
\hline \multirow[t]{2}{*}{ [18] } & Dabrafenib + trametinib & III & 11.4 & $72 \%$ at 1 year & 64 \\
\hline & Vemurafenib & & 7.3 & $65 \%$ at 1 year & 51 \\
\hline \multirow{2}{*}{$\begin{array}{l}{[19]} \\
{[20]}\end{array}$} & Cobimetinib + vemurafenib & $|/| \mid$ & Vemurafenib refractory: 2.8 & 8.4 months & 15 \\
\hline & & & Vemurafenib naïve: 13.7 & 28.5 months & 87 \\
\hline \multirow{2}{*}{$\begin{array}{l}{[21]} \\
{[22]}\end{array}$} & Cobimetinib + vemurafenib & III & 12.3 & $81 \%$ at 9 months & 70 \\
\hline & Vemurafenib & & 7.3 & $73 \%$ at 9 months & 50 \\
\hline [23] & Encorafenib + binimetinib & $|/| \mid$ & 11.3 & NA & 74.5 \\
\hline \multirow[t]{2}{*}{ [24] } & Binimetinib & III & NA & NA & 15 \\
\hline & DTIC & & & & 7 \\
\hline
\end{tabular}


with chemotherapy group. PFS rates at 6 months were $34 \%$ in pembrolizumab $2 \mathrm{mg} / \mathrm{kg}$ group, $38 \%$ in the $10 \mathrm{mg} / \mathrm{kg}$ group, and $16 \%$ in the chemotherapy group. Treatment-related grade 3-4 AEs were less in pembolizumab treatment groups, $11 \%$ for $2 \mathrm{mg} / \mathrm{kg}$ group and $14 \%$ for $10 \mathrm{mg} / \mathrm{kg}$ group, compared with $26 \%$ in the chemotherapy group. Again noted in the pembrolizumab groups, the most common treatment-related grade 3-4 AE was fatigue $(1 \%$ in the $2 \mathrm{mg} / \mathrm{kg}$ group and $<1 \%$ in the $10 \mathrm{mg} / \mathrm{kg}$ group, compared with $5 \%$ in the chemotherapy group). A follow-up pooled analysis of the 655 enrolled KEYNOTE-001 patients (135 from a nonrandomized cohort [ $\mathrm{n}=87$ ipilimumab naive; $\mathrm{n}=48$ ipilimumab treated] and 520 from randomized cohorts [ $\mathrm{n}=226$ ipilimumab naive; $\mathrm{n}=294$ ipilimumab treated $]$ ) was reported in 2016 [27]. Among the 581 patients with measurable disease at baseline, ORR was 33 with $45 \%$ in treatment-naive patients. $44 \%(90 / 205)$ of patients had response duration for at least 1 year and $79 \%(162 / 205)$ had response duration for at least 6 months. Median OS in the total population was 23 months with a 24-month survival rate of $49 \%$. In treatment-naive patients, median OS was 31 months with a 24-month survival rate of $60 \%$. It was further noted that a smaller tumor size at baseline was associated with a higher ORR. There was no significant difference in antitumor activity among the various pembrolizumab doses in randomized cohorts. The study results were recently updated to include 3 year overall survival data [28]. The 36-month OS rate was $40 \%$ and median OS was 23.8 months. Based on the finding of significant activity of pembrolizumab in treatment naïve patients, a randomized controlled phase III study was performed (KEYNOTE 006) enrolling 834 patients with advanced melanoma assigned in a 1:1:1 ratio to pembrolizumab $10 \mathrm{mg} / \mathrm{kg}$ every 2 weeks or every 3 weeks or four doses of ipilimumab $3 \mathrm{mg} / \mathrm{kg}$ every 3 weeks [29]. The estimated 6-month PFS rates were $47.3 \%$ for pembrolizumab every 2 weeks, $46.4 \%$ for pembrolizumab every 3 weeks, and $26.5 \%$ for ipilimumab (HR 0.58; p $<0.001$ for both pembrolizumab regimens versus ipilimumab; CIs $0.46-0.72$ and $0.47-0.72$, respectively). Estimated 12 -month survival rates were $74.1,68.4$, and $58.2 \%$, respectively (HR for pembrolizumab every 2 weeks, 0.63 ; $95 \%$ CI $0.47-0.83 ; \mathrm{p}=0.0005$; HR for pembrolizumab every 3 weeks, $0.69 ; 95 \%$ CI $0.52-0.90 ; \mathrm{p}=0.0036)$. Significant improvement in ORR were observed in both pembrolizumab treatment groups $(33.7 \%$ for every 2 weeks and $32.9 \%$ for every 3 weeks) compared with ipilimumab group (11.9 \%) ( $<<0.001$ for both comparisons). Reduced rates of treatment-related AEs of grade 3 to 5 severity were noted in the pembrolizumab groups (13.3 and $10.1 \%)$ compared with ipilimumab group
(19.9\%). Based on the fewer toxicities and significantly improved OS in the pembrolizumab treatment groups compared with ipilimumab group, FDA approved pembrolizumab as first-line therapy for metastatic melanoma. At a recent update with median follow-up duration of 22.9 months, the improvement in OS, PFS, and ORR with pembrolizumab over ipilimumab held up [30]. Median OS was not reached for either pembrolizumab groups versus 16.0 months with ipilimumab. The estimated 24-month OS rates were $55 \%$ for pembrolizumab group and $43 \%$ for ipilimumab group.

Nivolumab, a fully human IgG4 monoclonal antibody, was the first anti-PD-1 antibody to be evaluated in humans in a phase I trial [31]. Nivolumab at doses of $0.1-10 \mathrm{mg} / \mathrm{kg}$ produced a $31 \%$ response rate in 107 previously treated ipilimumab-naive metastatic melanoma patients in a phase I trial [6]. Median duration of response and median OS were 22 months and 17.3 months, respectively. Two subsequent randomized trials comparing nivolumab with chemotherapy have been reported in ipilimumab refractory patients and in untreated patients without BRAF mutation [32, 33]. Metastatic melanoma patients without BRAF mutation $(\mathrm{n}=418)$ were randomized to nivolumab $3 \mathrm{mg} /$ $\mathrm{kg}$ every 2 weeks or dacarbazine $1000 \mathrm{mg} / \mathrm{m}^{2}$ every 3 weeks as first line therapy [32]. Compared with dacarbazine as first-line therapy, nivolumab improved 1 -year OS rate, (72.9 versus $42.1 \%$; HR 0.42; $99.79 \%$ CI, 0.25-0.73; $\mathrm{p}<0.001$ ), median PFS (5.1 versus 2.2 months; HR 0.43; $95 \%$ CI $0.34-0.56$; p < 0.001) and ORR (40.0 versus $13.9 \%$; odds ratio 4.06; $\mathrm{p}<0.001$ ). Rates of common AEs associated with nivolumab such as fatigue, pruritus, and nausea were consistent with findings from phase I trials. Grade 3 or 4 AEs occurred in $11.7 \%$ of the nivolumab group and $17.6 \%$ of the dacarbazine group.

For ipilimumab refractory patients, nivolumab was also found to be superior to chemotherapy [33]. Patients who progressed after ipilimumab or ipilimumab and a BRAF inhibitor if they were BRAF V600 mutation-positive were randomized 2:1 to nivolumab $3 \mathrm{mg} / \mathrm{kg}$ every 2 weeks $(n=272)$ or investigator choice chemotherapy $(n=133)$. An ORR of $31.7 \%$ was observed in the nivolumab group compared with $10.6 \%$ in the chemotherapy group. There was no significant difference in response to nivolumab in patients with or without previous benefit from antiCTLA4 therapy, ORR of 30 and $32.5 \%$, respectively. This dataset has led to the FDA approval of single-agent nivolumab in ipilimumab-refractory metastatic melanoma patients in December 2014.

The studies reviewed above are briefly summarized in Table 2. 
Table 2 Recent checkpoint inhibitor study results in melanoma

\begin{tabular}{|c|c|c|c|c|c|c|}
\hline Study & Phase of study & Prior Tx & Agent & ORR (\%) & PFS & OS \\
\hline$[25]$ & I & Yes/no & Pembrolizumab & $31-51$ & $>7$ months & NA \\
\hline [5] & 1 & Yes & Pembrolizumab & 26 & $37-45 \%$ at 6 months & $58-63 \%$ at 1 year \\
\hline \multirow[t]{2}{*}[26]{} & $\|$ & Yes & Pembrolizumab & $21-25$ & $34-38 \%$ at 6 months & NA \\
\hline & & & Chemotherapy & 4 & $16 \%$ at 6 months & \\
\hline $\begin{array}{l}{[27]} \\
{[28]}\end{array}$ & $|/| \mid$ & Yes/no & Pembrolizumab & 33 & $35 \%$ at 1 year & $\begin{array}{l}23 \text { months } \\
40 \% \text { at } 3 \text { years }\end{array}$ \\
\hline \multirow{2}{*}{$\begin{array}{l}{[29]} \\
{[30]}\end{array}$} & III & No & Pembrolizumab & $32.9-33.7$ & $46.4-47.3 \%$ at 6 months & $55 \%$ at 2 years \\
\hline & & & Ipilimumab & 11.9 & $26.5 \%$ at 6 months & $43 \%$ at 2 years \\
\hline$[6]$ & 1 & Yes & Nivolumab & 31 & $27 \%$ at 2 years & $\begin{array}{l}16.8 \text { months } \\
43 \% \text { at } 2 \text { years }\end{array}$ \\
\hline \multirow[t]{2}{*}[32]{} & III & No & Nivolumab & 40.0 & 5.1 months & $72.9 \%$ at 1 year \\
\hline & & & DTIC & 13.9 & 2.2 months & $42.1 \%$ at 1 year \\
\hline \multirow[t]{2}{*}{ [33] } & 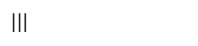 & Yes & Nivolumab & 31.7 & $48 \%$ at 6 months & NA \\
\hline & & & Chemotherapy & 10.6 & $34 \%$ at 6 months & \\
\hline
\end{tabular}

Prior Tx patients with prior treatment for metastatic melanoma included. Yes/No indicates a mixed population of treatment naïve and pre-treated patients

\section{Combination of checkpoint inhibitors}

The success of CTLA-4 inhibition and PD-1/PD-L1 inhibition in the treatment of cancer has lead to greater appreciation of the complexity of tumor microenvironment and the various interacting components that may present as unique opportunities for manipulation to control cancer. Conventional activated CD4+ and CD8+ $\mathrm{T}$ cells expressed CTLA-4 on the surfaces following induction [34, 35]. CTLA-4 binds to B7.1 (CD80) and B7.2 (CD86) on antigen presenting cells (APCs), where it competes with costimulatory receptor CD28. Binding of CTLA-4 to CD80/CD86 reduces CD28-dependent costimulation. CTLA-4 also mediates direct inhibitory effects on the MHC-TCR pathway by impairing TCR signaling [36]. Furthermore, CTLA-4 is constitutively expressed on CD4+CD25+FOXP3+ regulatory $\mathrm{T}$ cells and plays a role in their suppressive functions [37-39]. Programmed cell death protein-1 (PD-1; also known as CD27) is also a coinhibitory CD28-family molecule [35]. While CTLA-4 functions in the early phase of naive $\mathrm{T}$ cell activation, PD-1 is mainly active in the late phase by inducing exhaustion in effector T cells. PD-1 is expressed on activated $\mathrm{T}$ cells, $\mathrm{T}$ regs [40], activated $\mathrm{B}$ cells, NK cells, and monocytes. It binds to PD-L1 (programmed death ligand-1, B7-H1) and PD-L2 (programmed death ligand-2, B7-DC) on APCs. PD-1 binding results in decreased TCR signaling [36]. Tumor cells utilize the PD1-PD-L1/2 pathway to evade immune surveillance [41]. The observation that PD-1 inhibition is active in CTLA-4 inhibitor refractory patients confirms the complementary effects of dual checkpoint inhibition [5,33].

In a randomized double-blind 3-arm study, treatment naive unresectable stage III or IV melanoma patients $(\mathrm{n}=945)$ were randomized in a 1:1:1 ratio to nivolumab $3 \mathrm{mg} / \mathrm{kg}$ every 2 weeks, nivolumab $1 \mathrm{mg} / \mathrm{kg}$ every 3 weeks plus ipilimumab $3 \mathrm{mg} / \mathrm{kg}$ every 3 weeks for four doses followed by nivolumab $3 \mathrm{mg} / \mathrm{kg}$ every 2 weeks for cycle three and beyond, or ipilimumab $3 \mathrm{mg} / \mathrm{kg}$ every 3 weeks for four doses [42]. The median PFS was 11.5 months in the combination group compared with 2.9 months in the ipilimumab group (HR 0.42; $99.5 \% \mathrm{CI}, 0.31-0.57$; $\mathrm{p}<0.001$ ) and 6.9 months in the nivolumab group (HR for the comparison with ipilimumab, 0.57; $99.5 \% \mathrm{CI}$, $0.43-0.76 ; \mathrm{p}<0.001)$. While median PFS was similar between the combination group and the nivolumab group in patients with tumors positive for the PD-1 ligand (PD-L1) at 14.0 months, median PFS was longer with the combination therapy than with nivolumab alone in patients with PD-L1-negative tumors (11.2 versus 5.3 months). The activity of nivolumab-ipilimumab combination was similar in patients with and without BRAF mutation. ORR were $43.7 \%$ in the nivolumab group, $57.6 \%$ in the combination group, and $19.0 \%$ in the ipilimumab group. The percentage of CR was higher in the combination group $(11.5 \%)$ than in either the nivolumab group $(8.9 \%)$ or the ipilimumab group $(2.2 \%)$. More treatment related AEs of grade 3 or 4 occurred in the combination group $(55.0 \%)$ compared with those of the nivolumab group $(16.3 \%)$ and the ipilimumab group (27.3\%). Discontinuation of treatment due to AEs also occurred more frequently in the combination group (36.4\%) compared with nivolumab group $(7.7 \%)$ and ipilimumab group (14.8\%). While one study-drug related death was reported in the nivolumab group (neutropenia) and one in the ipilimumab group (cardiac arrest), none was reported in the combination group. The study results 
were updated after more than 18 months of follow-up [43]. Median PFS continued to be significantly longer for combination group (11.5 months) and nivolumab group (6.9 months) compared with ipilimumab group (2.9 months) ( $\mathrm{p}<0.001)$. Median duration of response for combination group responders has not been reached compared with 22.3 months for the nivolumab responders and 14.4 months for the ipilimumab responders.

The improvement in tumor response with combination CTLA-4 and PD-1 inhibition compared with CTLA-4 inhibition alone was also observed in another randomized double-blind trial [44]. Treatment naïve metastatic melanoma patients $(n=142)$ were randomly assigned in a $2: 1$ ratio to ipilimumab $3 \mathrm{mg} / \mathrm{kg}$ and nivolumab $1 \mathrm{mg} / \mathrm{kg}$ or placebo once every 3 weeks for four doses, followed by nivolumab $3 \mathrm{mg} / \mathrm{kg}$ or placebo every 2 weeks. Among patients with BRAF wild-type tumors, ORR was $61 \%$ in the combination group versus $11 \%$ in the monotherapy $(\mathrm{p}<0.001)$. There were 16 patients $(22 \%)$ with $\mathrm{CR}$ in the combination group and none in the monotherapy group. Similar findings were also observed among patients with BRAF mutationpositive tumors with ORR of $52 \%$ for those receiving combination therapy and CR rate of $22 \%$. Similar to the observation in the other randomized trial, the combination therapy is associated with more frequent grade 3 or 4 drug-related AEs compared with monotherapy group, 54 versus $24 \%$. The most common grade 3 or 4 AEs associated with the combination therapy were colitis (17\%), diarrhea $(11 \%)$, and an elevated alanine aminotransferase level $(11 \%)$. However, there was no significant difference in response rates between patients whose pretreatment tumors were defined as PD-L1-positive and those whose tumors were PD-L1-negative. Despite the significant clinical efficacy with combination of nivolumab and ipilimumab demonstrated in these initial studies, caution was raised for further studies to clarify the various patient subsets with optimal upfront therapy [45].

The impressive efficacy with dual CTLA-4 and PD-1 inhibition was also reported in the KEYNOTE-029 expansion trial testing pembrolizumab and ipilimumab combination [46]. Treatment naïve metastatic melanoma patients $(\mathrm{n}=153)$ received pembrolizumab $2 \mathrm{mg} / \mathrm{kg}$ every 3 weeks plus ipilimumab $1 \mathrm{mg} / \mathrm{kg}$ every 3 weeks for 4 doses followed by pembrolizumab $2 \mathrm{mg} /$ kg every 3 weeks until intolerable toxicity, progression, or 24 months. Grade 3-4 treatment related AEs were observed in 41 patients (38\%). ORR by central review was $57 \%$ with $10 \% \mathrm{CR}$ and $47 \%$ partial responses (PR). PFS at 6 months was $70 \%$.

The studies reviewed above are briefly summarized in Table 3.

\section{Oncolytic virus}

Another promising novel therapy of melanoma is the recently approved genetically modified oncolytic virus for intralesional injection of cutaneous, subcutaneous, and nodal metastatic melanoma patients who progressed after surgery. Oncolytic virus preferentially replicates in tumor cells which results in tumor cell lysis. Following cell lysis, tumor-associated antigens and danger-associated molecules were released to promote anti-tumor immune responses as well as release of new viral particles for infecting nearby viable tumor cells. In the case of the recently approved talimogene laherparepvec (T-VEC), anti-tumor immune response following oncolysis is putatively enhanced by viral expression of GM-CSF which was inserted into the T-VEC genome. T-VEC is a Herpes simplex virus type- 1 with several key genetic modifications. Deletion of ICP34.5 gene results in enhanced viral replication in cancer cells [47]. Deletion of ICP47 enhances antigen presentation and maintains cell surface MHC-I-antigen expression on infected cancer cells [48, 49]. Further improving the immunogenicity of T-VEC, 2 copies of the human GM-CSF gene were incorporated into the deleted ICP34.5 genome location [50].

In a phase I study, T-VEC has been to shown to replicate intratumorally and express GM-CSF with acceptable safety profile [51]. In phase II study, ORR of $26 \%(\mathrm{n}=13)$ was observed in 50 stage IIIc and IV melanoma patients

Table 3 Recent combination checkpoint inhibitor study results in melanoma

\begin{tabular}{|c|c|c|c|c|c|}
\hline Study & Phase of study & Prior Tx & Agents & ORR & PFS \\
\hline \multirow{3}{*}{$\begin{array}{l}{[42]} \\
{[43]}\end{array}$} & \multirow[t]{3}{*}{ III } & \multirow[t]{3}{*}{ No } & Ipilimumab & 19.0 & 2.9 months \\
\hline & & & Nivolumab & 43.7 & 6.9 months \\
\hline & & & Nivolumab + ipilimumab & 57.6 & 11.5 months \\
\hline \multirow[t]{2}{*}[44]{} & \multirow[t]{2}{*}{ III } & \multirow[t]{2}{*}{ No } & Nivolumab + ipilimumab & $52-61$ & $\begin{array}{l}8.5 \text { months (BRAF mutant) } \\
\text { Not reached (BRAF wildtype) }\end{array}$ \\
\hline & & & Ipilimumab & 11 & $\begin{array}{l}2.7 \text { months (BRAF mutant) } \\
4.4 \text { months (BRAF wildtype) }\end{array}$ \\
\hline$[46]$ & $\|$ & No & Pembrolizumab + ipilimumab & 57 & $70 \%$ at 6 months \\
\hline
\end{tabular}


with 8 CRs and 5 PRs [52]. Responses were observed in both injected and uninjected lesions, including visceral lesions. In a phase III multicenter trial, 436 patients with unresectable stage IIIB-IV melanoma patients were randomized at a 2:1 ratio to intralesional T-VEC $(n=295)$ or subcutaneous GM-CSF $(\mathrm{n}=141)$ [53]. Durable response rates (DRR; responses lasting 6 months or more) was significantly higher in the T-VEC group (16.3\%; $95 \%$ CI 12.1-20.5 \%) compared with GM-CSF control group (2.1 \%; 95 \% CI 0-4.5\%; odds ratio, 8.9; $\mathrm{p}=0.001)$. ORR in the T-VEC arm was higher at $26.4 \%$ compared with $5.7 \%$ in the control arm. Although there was a trend for longer median OS in the T-VEC arm at 23.3 months compared with 18.9 months in the control arm, it was not significant $(\mathrm{p}=0.051)$. The most common AEs with T-VEC were fatigue, chills, and pyrexia. The most common grade 3 or $4 \mathrm{AE}$ was cellulitis (2.1\%). This dataset led to the FDA approval of T-VEC for intratumoral injection of cutaneous, subcutaneous, and nodal metastatic melanoma. To further elucidate the immunologic basis of its mechanism of action, a post hoc analysis of the phase II study $(\mathrm{n}=50)$ was performed [54]. Reduction of tumor burden $\geq 30 \%$ of uninjected non-visceral lesions was observed in 11 of 23 patients $(47.8 \%)$ and $\geq 30 \%$ reduction in the total burden of visceral lesions was observed in 2 of 12 patients ( $16.7 \%)$.

\section{Multimodal combination therapy}

With the promising activity of both RAS-RAF-MAPK pathway inhibition and checkpoint inhibition in melanoma treatment, combination of both modes of therapy has been attempted. BRAF inhibition can have immunosensitization effects through increased antigen presentation, [55-57]. Antigen-specific T cell recognition [56, 58], homing of immune effector cell to the tumors [57, 59, $60]$ and improved $T$ cell effector functions [61, 62]. MEK inhibition can also have immunosensitizing effect via upregulation of tumor antigen expression and presentation $[55,63]$. Paradoxically, MEK inhibitor can also dampen immune effector functions since impaired $\mathrm{T}$ cell proliferation and functions with MEK inhibition have been demonstrated in vitro [55, 64].

However, significant liver toxicity resulting in trial termination was observed in the first clinical trial of vemurafenib plus ipilimumab [65]. The first cohort of six patients received a run-in period of 1 month of vemurafenib (960 mg BID) only, followed by ipilimumab ( $3 \mathrm{mg} / \mathrm{kg}$ every 3 weeks for four doses) and concurrent BID doses of vemurafenib. Grade 3 elevations in aminotransferase levels developed in four patients following the first infusion of ipilimumab plus vemurafenib. The first 4 of 6 patients enrolled in the second cohort received a lower dose of vemurafenib (720 mg BID) plus the full dose of ipilimumab. Elevations in aminotransferase levels (grade 3 in two patients and grade 2 in one patient) developed within 3 weeks after starting ipilimumab. The remaining two patients in the second cohort received vemurafenib alone.

In a study combining dabrafenib with or without trametinib and ipilimumab at doses of dabrafenib $100 \mathrm{mg}$ $\mathrm{BID}$, trametinib $1 \mathrm{mg}$ QD and ipilimumab $3 \mathrm{mg} / \mathrm{kg}$ every 3 weeks for 4 doses, grade 3 colitis complicated by perforation was observed in 2 of 7 advanced melanoma patients [66]. The enrollment to the triple combination arm was stopped with ongoing enrollment to the dabrafenib and ipilimumab combination arm. Another ongoing phase I trial is evaluating the combination of an anti-PD-L1 antibody MEDI4736 (durvolumab) at $10 \mathrm{mg} / \mathrm{kg}$ every 2 weeks plus dabrafenib $150 \mathrm{mg}$ BID and trametinib $2 \mathrm{mg}$ QD [67]. Patients were stratified by BRAF mutation status into three different cohorts. BRAF-mutant patients received the triple combination and BRAF wild-type patients received durvolumab plus trametinib or sequential trametinib then durvolumab. In the BRAF mutant cohort, treatment with the triple combination resulted in an ORR of $69 \%$, and DCR of $100 \%$. In the BRAF WT cohorts, ORR and DCR were 21 and $79 \%$, respectively, in the combination group and 13 and $80 \%$, respectively, in the sequential group. There was no significant increase in immune related AEs reported to date.

T-VEC and ipilimumab combination was also tested in an open-label, multicenter phase Ib study [68]. Patients $(\mathrm{n}=19)$ with unresectable stage IIIB-IVM1c melanoma with injectable cutaneous, subcutaneous, or nodal lesion and without prior systemic therapy were enrolled. Intratumoral T-VEC as administered according to approved dosages and schedule. Beginning in week 6 , ipilimumab $(3 \mathrm{mg} / \mathrm{kg})$ was administered every 3 weeks for four infusions. One patient received one dose of T-VEC and withdrew consent. The efficacy analysis included 18 patients. Five patients $(26.3 \%)$ reported grade 3 or higher treatment-related AEs, with three patients $(15.8 \%)$ attributable to T-VEC and four patients (21.1\%) attributable to ipilimumab. ORR was $50 \%$ with four patients having confirmed CR (22\%). Median time to response was 5.3 months (range, 2.6-8.1 months). DRR was $44 \%$. Responses were seen in both injected and uninjected lesions. Among the 35 injected lesions, $26(74 \%)$ regressed $\geq 50 \%$ and $11(31 \%)$ regressed completely. Among the 23 uninjected measurable lesions, 12 (52\%) regressed $\geq 50 \%$, and nine (39\%) regressed completely. Regression of uninjected non-visceral lesions was observed in five of $10(50 \%)$ measurable visceral lesions with $\geq 50 \%$ regression. 


\section{Promising novel agents under development Glembatumumab vedotin}

The human 560-amino-acid type I glycoprotein NMB (gpNMB) is an intracellular transmembrane protein that transits the cell surface with homology to pMEL-17, a melanocyte specific marker $[69,70]$. It is over-expressed in melanoma [70]. Glembatumumab vedotin (CDX-011 or CR011-vcMMAE) was produced by covalently linking a fully human IGG2 monoclonal antibody against gpNMB (CR011) to monomethyl auristatin E (MMAE), a potent mitotic spindle formation inhibitor [71-73]. It is designed to deliver the MMAE payload upon binding to gpNMB on the tumor cells and releasing free MMAE following lysosomal internalization and proteolytic cleavage of the valine-citrulline linker. Cell death ensues after microtubule inhibition by the free MMAE. Glembatumumab vedotin was active against melanoma cell lines expressing gpNMB in vitro and in pre-clinical model $[70,74]$. A phase I/II study was conducted to assess the safety and activity of glembatumumab vedotin in patients with unresectable stage III or stage IV melanoma [75]. Enrolled patients $(\mathrm{n}=117)$ received glembatumumab vedotin at 3 dosing schedules: every 3 weeks (schedule $1, \mathrm{n}=79$ ), 2 of 3 weeks (schedule $2, \mathrm{n}=15$ ), and weekly (schedule $3, \mathrm{n}=23$ ) at escalating dosages. Grade $3 / 4$ treatment-related AEs occurring in $\geq 2$ patients were rash, neutropenia, fatigue, neuropathy, arthralgia, myalgia, and diarrhea. Three treatment-related deaths (resulting from pneumococcal sepsis, toxic epidermal necrolysis, and renal failure) occurred at doses exceeding the MTDs. In the schedule 1 phase II expansion cohort $(\mathrm{n}=34)$, there were five patients $(15 \%)$ with PR and 8 (24\%) with stable disease (SD) for 6 months. ORR was 2 of 6 (33\%) for schedule 2 MTD and 3 of 12 (25\%) for the schedule 3 MTD.

\section{Pv-10}

PV-10 is a sterile preparation of $10 \%$ solution of rose bengal disodium (RB) in $0.9 \%$ saline for intralesional injection into tumors [76]. RB has been used as intravenous diagnostic dye for liver function and as topical solution for ophthalmic conditions [77, 78]. Following intratumor injection, PV-10 accumulates in tumor lysosomes resulting in rapid lysis of tumor cells [79]. The lysis of tumor cells following PV-10 injection also induces tumor-specific $\mathrm{T}$ cell-mediated immune response with regression of uninjected lesions [80, 81]. In a phase 1 study, 8 patients with dermal and/or subcutaneous metastatic melanoma were enrolled [81]. CR was observed in injected and uninjected lesions in 4 of the 8 patients. All 8 patients exhibited partial to complete regression of the injected lesion. Best overall response rate (BORR) at 12 weeks is $55 \%$. Of the 6 enrolled patients with history of prior ipilimumab, PD-1 inhibitor, and/or vemurafenib trestments, 4 of 6 exhibited CR of the injected and uninjected lesions. In a subsequent multicenter phase II study, 62 stage III and IV treatment-refractory patients with at least 1 cutaneous or subcutaneous lesion were enrolled [82]. Intralesional (IL) injection of PV-10 at $0.5 \mathrm{ml}$ per $\mathrm{cm}^{3}$ lesion volume was administered into each measurable lesion up to 20 study lesions. Treatments were repeated at weeks 8,12 , and 16 for new non-target lesions or existing target or non-target lesions not exhibiting complete response. BORR of target lesions was $51 \%$ with $26 \%$ CR and $25 \%$ PR. DCR was achieved in $69 \%$ of patients. Median time to response was 1.9 months. The median duration of response was 4.0 months. On analysis of lesion-specific responses of the 491 target lesions, CR, PR and SD were 53, 5, and $12 \%$, respectively. $26 \%$ of 42 patients with designated bystander lesions experienced complete regression of the uninjected bystander lesions. Treatment was well-tolerated with predominantly mild to moderate locoregional or injection site-related AEs.

\section{Conclusion}

The treatment paradigm for the management of advanced metastatic melanoma patients continues to shift with the rapid development of effective agents and combination therapy. With the many available active anti-melanoma agents, the optimal treatment algorithm for various subsets of patients, e.g. BRAF mutants, NRAS mutants, PD-1 expressing, has yet to be determined. With further understanding of key pathway molecules in melanoma oncogenesis, elucidation of components of tumor microenvironment, fine-tuning of drivers in the host-tumor immune relationship, and introduction of novel antitumor agents $[83,84]$, the outcome for metastatic melanoma will continue to improve.

\section{Authors' contributions}

ECH prepared the majority of the manuscript. KCG performed the literature compilation was a major contributor in preparation of the manuscript. All authors read and approved the final manuscript.

\section{Acknowledgements \\ Not applicable. \\ Competing interests \\ $\mathrm{ECH}$ is on the Speaker Bureau of Amgen, Inc, and receives Honoria for speak- ing engagement. \\ Funding \\ ECH receives research support from Saint Louis University Cancer Center.}

Received: 2 July 2016 Accepted: 2 August 2016

Published online: 08 August 2016 


\section{References}

1. Hodi FS, O'Day SJ, McDermott DF, Weber RW, Sosman JA, Haanen JB, et al. Improved survival with ipilimumab in patients with metastatic melanoma. N Engl J Med. 2010;363(8):711-23.

2. Chapman P, Hauschild A, Robert C, Haanen J, Ascierto P, Larkin J, et al. Improved survival with vemurafenib in melanoma with BRAF V600E mutation. N Engl J Med. 2011;364(26):2507-16.

3. Ascierto P, Minor D, Ribas A, Lebbe C, O'Hagan A, Arya N, et al. Phase II trial (BREAK-2) of the BRAF inhibitor dabrafenib (GSK2118436) in patients with metastatic melanoma. J Clin Oncol. 2013;31(26):3205-11.

4. Flaherty KT, Infante JR, Daud A, Gonzalez R, Kefford RF, Sosman J, et al. Combined BRAF and MEK inhibition in melanoma with BRAF V600 mutations. N Engl J Med. 2012;367(18):1694-703.

5. Robert C, Ribas A, Wolchok JD, Hodi FS, Hamid O, Kefford R, et al. Antiprogrammed-death-receptor-1 treatment with pembrolizumab in ipilimumab-refractory advanced melanoma: a randomised dose-comparison cohort of a phase 1 trial. Lancet. 2014;384(9948):1109-17.

6. Topalian SL, Sznol M, McDermott DF, Kluger HM, Carvajal RD, Sharfman WH, et al. Survival, durable tumor remission, and long-term safety in patients with advanced melanoma receiving nivolumab. J Clin Oncol. 2014;32(10):1020-30.

7. Davies H, Bignell G, Cox C, Stephens P, Edkins S, Clegg S, et al. Mutations of the BRAF gene in human cancer. Nature. 2002;417(6892):949-54.

8. Hauschild A, Grob JJ, Demidov LV, Jouary T, Gutzmer R, Millward M, et al. Dabrafenib in BRAF-mutated metastatic melanoma: a multicentre, open-label, phase 3 randomised controlled trial. Lancet. 2012;380(9839):358-65.

9. Sosman JA, Kim KB, Schuchter L, Gonzalez R, Pavlick AC, Weber JS, et al. Survival in BRAF V600-mutant advanced melanoma treated with vemurafenib. N Eng J Med. 2012;366(8):707-14.

10. Shi H, Hugo W, Kong X, Hong A, Koya RC, Moriceau G, et al. Acquired resistance and clonal evolution in melanoma during BRAF inhibitor therapy. Cancer Discov. 2014;4(1):80-93.

11. Rizos H, Menzies AM, Pupo GM, Carlino MS, Fung C, Hyman J, et al. BRAF inhibitor resistance mechanisms in metastatic melanoma: spectrum and clinical impact. Clin Cancer Res. 2014;20(7):1965-77.

12. Wagle N, Emery C, Berger MF, Davis MJ, Sawyer A, Pochanard P, et al. Dissecting therapeutic resistance to RAF inhibition in melanoma by tumor genomic profiling. J Clin Oncol. 2011;29(22):3085-96.

13. Su F, Viros A, Milagre C, Trunzer K, Bollag G, Spleiss O, et al. RAS mutations in cutaneous squamous-cell carcinomas in patients treated with BRAF inhibitors. N Engl J Med. 2012;366(3):207-15.

14. King AJ, Arnone MR, Bleam MR, Moss KG, Yang J, Fedorowicz KE, et al. Dabrafenib: preclinical characterization, increased efficacy when combined with trametinib, while BRAF/MEK tool combination reduced skin lesions. PLoS ONE. 2013;8(7):e67583.

15. Flaherty KT, Robert C, Hersey P, Nathan P, Garbe C, Milhem M, et al. Improved survival with MEK inhibition in BRAF-mutated melanoma. N Eng J Med. 2012;367(2):107-14.

16. Long GV, Stroyakovskiy D, Gogas H, Levchenko E, de Braud F, Larkin J, et al. Combined BRAF and MEK inhibition versus BRAF inhibition alone in melanoma. N Engl J Med. 2014;371(20):1877-88.

17. Long GV, Stroyakovskiy D, Gogas H, Levchenko E, de Braud F, Larkin J, et al. Dabrafenib and trametinib versus dabrafenib and placebo for Val600 BRAF-mutant melanoma: a multicentre, double-blind, phase 3 randomised controlled trial. Lancet. 2015;386(9992):444-51.

18. Robert C, Karaszewska B, Schachter J, Rutkowski P, Mackiewicz A, Stroiakovski D, et al. Improved overall survival in melanoma with combined dabrafenib and trametinib. N Engl J Med. 2015;372(1):30-9.

19. Ribas A, Gonzalez R, Pavlick A, Hamid O, Gajewski TF, Daud A, et al. Combination of vemurafenib and cobimetinib in patients with advanced BRAF(V600)-mutated melanoma: a phase 1b study. Lancet Oncol. 2014;15(9):954-65.

20. Pavlick AC, Ribas A, Gonzalez R, Hamid O, Gajewski T, Daud A, Puzanov I, Hsu JJ, Choong NW, McArthur GA. Extended follow-up results of phase lb study (BRIM7) of vemurafenib (VEM) with cobimetinib (COBI) in BRAFmutant melanoma. J Clin Oncol. 2015;33(15):9020.

21. Larkin J, Ascierto PA, Dréno B, Atkinson V, Liszkay G, Maio M, et al. Combined vemurafenib and cobimetinib in BRAF-mutated melanoma. N Engl J Med. 2014;371(20):1867-76.
22. Larkin JMG, Yan Y, Mcarthur GA, Ascierto PA, Liszkay G, Maio M, et al. Update of progression free survival (PFS) and correlative biomarker analysis from coBRIM: phase III study of cobimetinib (cobi) plus vemurafenib (vem) in advanced BRAF-mutated melanoma. J Clin Oncol. 2015;33:9006.

23. Sullivan RJ, Weber JS, Patel SP, Dummer R, Miller WH, Cosgrove D, et al. A phase Ib/II study of BRAF inhibitor (BRAFi) encorafenib (ENCO) plus MEK inhibitor (MEKi) binimetinib (BINI) in cutaneous melanoma patients naive to BRAFi treatment. J Clin Oncol. 2015;33:9007.

24. Dummer R, Schadendorf D, Ascierto PA, Arance Fernández AM, Dutriaux C, Maio M, Rutkowski P, et al. Results of NEMO: A phase III trial of binimetinib (BINI) vs dacarbazine (DTIC) in NRAS-mutant cutaneous melanoma. J Clin Oncol. 2016;34:9500.

25. Hamid O, Robert C, Daud A, Hodi FS, Hwu WJ, Kefford R, et al. Safety and tumor responses with lambrolizumab (anti-PD-1) in melanoma. N Engl J Med. 2013;369(2):134-44.

26. Ribas A, Puzanov I, Dummer R, Schadendorf D, Hamid O, Robert C, et al. Pembrolizumab versus investigator-choice chemotherapy for ipilimumab-refractory melanoma (KEYNOTE-002): a randomised, controlled, phase 2 trial. Lancet Oncol. 2015;16:908-18.

27. Ribas A, Hamid O, Daud A, Hodi FS, Wolchok JD, Kefford R, et al. Association of pembrolizumab with tumor response and survival among patients with advanced melanoma. JAMA. 2016:315(15):1600-9.

28. Robert C, Ribas A, Hamid O, Daud A, Wolchok JD, Joshua AM, et al. Threeyear overall survival for patients with advanced melanoma treated with pembrolizumab in KEYNOTE-001. J Clin Oncol. 2016;34:9503.

29. Robert C, Schachter J, Long GV, Arance A, Grob JJ, Mortier L, et al. Pembrolizumab vs ipilimumab in advanced melanoma. N Engl J Med. 2015;372(26):2521-32.

30. Schachter J, Ribas A, Long GV, Arance A, Grob JJ, Mortier L, et al. Pembrolizumab versus ipilimumab for advanced melanoma: final overall survival analysis of KEYNOTE-006. J Clin Oncol. 2016;34:9504.

31. Brahmer JR, Drake CG, Wollner I, Powderly JD, Picus J, Sharfman WH, et al. Phase I study of single-agent anti-programmed death-1 (MDX-1106) in refractory solid tumors: safety, clinical activity, pharmacodynamics, and immunologic correlates. J Clin Oncol. 2010;28:3167-75.

32. Robert C, Long GV, Brady B, Dutriaux C, Maio M, Mortier L, et al. Nivolumab in previously untreated melanoma without BRAF mutation. N Engl J Med. 2015;372:320-30.

33. Weber JS, D'Angelo SP, Minor D, Hodi FS, Gutzmer R, Neyns B, et al. Nivolumab versus chemotherapy in patients with advanced melanoma who progressed after anti-CTLA-4 treatment (CheckMate 037): a randomised, controlled, open-label, phase 3 trial. Lancet Oncol. 2015;16:375-84.

34. Rudd CE, Taylor A, Schneider H. CD28 and CTLA-4 coreceptor expression and signal transduction. Immunol Rev. 2009;229(1):12-26.

35. Chen L. Co-inhibitory molecules of the B7-CD28 family in the control of T-cell immunity. Nat Rev Immunol. 2004;4(5):336-47.

36. Nirschl CJ, Drake CG. Molecular pathways: coexpression of immune checkpoint molecules: signaling pathways and implications for cancer immunotherapy. Clin Cancer Res. 2013;19(18):4917-24.

37. Sansom DM, Walker LS. The role of CD28 and cytotoxic T-lymphocyte antigen-4 (CTLA-4) in regulatory T-cell biology. Immunol Rev. 2006:212:131-48

38. Wing K, Onishi Y, Prieto-Martin P, Yamaguchi T, Miyara M, Fehervari Z, et al. CTLA-4 control over Foxp3 + regulatory T cell function. Science. 2008;322(5899):271-5.

39. Walker LSK. Treg and CTLA-4: two intertwining pathways to immune tolerance. J Autoimmun. 2013;45:49-57.

40. Francisco LM, Salinas VH, Brown KE, Vanguri VK, Freeman GJ, Kuchroo VK, et al. PD-L1 regulates the development, maintenance, and function of induced regulatory T cells. J Exp Med. 2009;206(13):3015-29.

41. Zou W, Chen L. Inhibitory B7-family molecules in the tumour microenvironment. Nat Rev Immunol. 2008;8(6):467-77.

42. Larkin J, Chiarion-Sileni V, Gonzalez R, Grob JJ, Cowey CL, Lao CD, et al. Combined nivolumab and ipilimumab or monotherapy in untreated melanoma N Engl J Med. 2015:373.23-34.

43. Wolchok JD, Chiarion-Sileni V, Gonzalez R, Rutkowski P, Grob JJ, Cowey CL, et al. Updated results from a phase III trial of nivolumab (NIVO) combined with ipilimumab (IPI) in treatment-naive patients (pts) with advanced melanoma (MEL) (CheckMate 067). J Clin Oncol. 2016;34:9505. 
44. Postow MA, Chesney J, Pavlick AC, Robert C, Grossmann K, McDermott $D$, et al. Nivolumab and ipilimumab versus ipilimumab in untreated melanoma. N Engl J Med. 2015;372:2006-17.

45. Tsai KK, Daud Al. Nivolumab plus ipilimumab in the treatment of advanced melanoma. J Hematol Oncol. 2015;31(8):123.

46. Long GV, Atkinson V, Cebon JS, Jameson MB, Fitzharris BM, McNeil CM, et al. Pembrolizumab (pembro) plus ipilimumab (ipi) for advanced melanoma: results of the KEYNOTE-029 expansion cohort. J Clin Oncol. 2016;34:9506.

47. Liu BL, Robinson M, Han ZQ, Branston RH, English C, Reay P, et al. ICP34.5 deleted herpes simplex virus with enhanced oncolytic, immune stimulating, and anti-tumour properties. Gene Ther. 2003;10:292-303.

48. Tomazin R, van Schoot NE, Goldsmith K, Jugovic P, Sempe P, Fruh K, et al. Herpes simplex virus type 2 ICP47 inhibits human TAP but not mouse TAP. J Virol. 1998;72:2560-3.

49. Hill A, Jugovic P, York I, Russ G, Bennink J, Yewdell J, et al. Herpes simplex virus turns off the TAP to evade host immunity. Nature. 1995;375:411-5.

50. Kaufman HL, Ruby CE, Hughes T, Slingluff CL Jr. Current status of granulocyte-macrophage colony-stimulating factor in the immunotherapy of melanoma. J Immunother Cancer. 2014:2:11.

51. Hu JCC, Coffin RS, Davis CJ. A phase I study of OncoVEXGM-CSF, a second-generation oncolytic herpes simplex virus expressing granulocyte macrophage colony-stimulating factor. Clin Cancer Res. 2006;12:6737-47.

52. Senzer NN, Kaufman HL, Amatruda T, Nemunaitis M, Reid T, Daniels G, et al. Phase II clinical trial of a granulocyte-macrophage colony stimulating factor-encoding, second-generation oncolytic herpesvirus in patients with unresectable metastatic melanoma. J Clin Oncol. 2009;27:5763-71.

53. Andtbacka RHI, Kaufman HL, Collichio F, Amatruda T, Senzer N, Chesney J, et al. Talimogene laherparepvec improves durable response rate in patients with advanced melanoma. J Clin Oncol. 2015;33:2780-8.

54. Kaufman HL, Amatruda T, Reid T, Gonzalez R, Glaspy J, Whitman E, et al. Systemic versus local responses in melanoma patients treated with talimogene laherparepvec from a multi-institutional phase II study. J Immunother Cancer. 2016;15(4):12.

55. Boni A, Cogdill AP, Dang P, Udayakumar D, Njauw CN, Sloss CM, et al. Selective BRAFV600E inhibition enhances T-cell recognition of melanoma without affecting lymphocyte function. Cancer Res. 2010;70(13):5213-9.

56. Sapkota B, Hill CE, Pollack BP. Vemurafenib enhances MHC induction in BRAF homozygous melanoma cells. Oncoimmunology. 2013;2:22890.

57. Frederick DT, Piris A, Cogdill AP, Cooper ZA, Lezcano C, Ferrone CR, et al. BRAF inhibition is associated with enhanced melanoma antigen expression and a more favorable tumor microenvironment in patients with metastatic melanoma. Clin Cancer Res. 2013;19(5):1225-31.

58. Donia M, Fagone P, Nicoletti F, Andersen RS, Høgdall E, Straten PT, et al. BRAF inhibition improves tumor recognition by the immune system: Potential implications for combinatorial therapies against melanoma involving adoptive T-cell transfer. Oncoimmunology. 2012;1(9):1476-83.

59. Wilmott JS, Long GV, Howle JR, Haydu LE, Sharma RN, Thompson JF, et al. Selective BRAF inhibitors induce marked T-cell infiltration into human metastatic melanoma. Clin Cancer Res. 2012;18(5):1386-94.

60. Long GV, Wilmott JS, Haydu LE, Tembe V, Sharma R, Rizos H, Thompson $J F$, et al. Effects of BRAF inhibitors on human melanoma tissue before treatment, early during treatment, and on progression. Pigment Cel Melanoma Res. 2013;26(4):499-508.

61. Koya RC, Mok S, Otte N, Blacketor KJ, Comin-Anduix B, Tumeh PC, Minasyan A, et al. BRAF inhibitor vemurafenib improves the antitumor activity of adoptive cell immunotherapy. Cancer Res. 2012;72(16):3928-37.

62. Comin-Anduix B, Chodon T, Sazegar H, Matsunaga D, Mock S, Jalil J, et al. The oncogenic BRAF kinase inhibitor PLX4032/RG7204 does not affect the viability or function of human lymphocytes across a wide range of concentrations. Clin Cancer Res. 2010;16(24):6040-8.

63. Kono M, Dunn IS, Durda PJ, Butera D, Rose LB, Haggerty TJ, et al. Role of the mitogen-activated protein kinase signaling pathway in the regulation of human melanocytic antigen expression. Mol Cancer Res. 2006:4(10):779-92.

64. Vella LJ, Pasam A, Dimopoulos N, Andrews M, Knights A, Puaux AL, et al. MEK inhibition, alone or in combination with BRAF inhibition, affects multiple functions of isolated normal human lymphocytes and dendritic cells. Cancer Immunol Res. 2014;2(4):351-60.
65. Ribas A, Hodi FS, Callahan M, Konto C, Wolchok J. Hepatotoxicity with combination of vemurafenib and ipilimumab. N Engl J Med. 2013;368:1365-6.

66. Puzanov I, Callahan MK, Linette GP, Patel SP, Luke JL, Sosman JA, et al. Phase 1 study of the BRAF inhibitor dabrafenib (D) with or without the MEK inhibitor trametinib (T) in combination with ipilimumab (Ipi) for V600E/K mutation-positive unresectable or metastatic melanoma (MM). J Clin Oncol. 2014;32(5):2511.

67. Ribas A, Butler M, Lutzky J, Lawrence DP, Robert C, Miller W, et al. Phase I study combining anti-PD-L1 (MEDI4736) with BRAF (dabrafenib) and/ or MEK (trametinib) inhibitors in advanced melanoma. J Clin Oncol. 2015;33:3003.

68. Puzanov I, Milhem MM, Minor D, Hamid O, Li A, Chen L, et al. Talimogene laherparepvec in combination with ipilimumab in previously untreated, unresectable stage IIIB-IV melanoma. J Clin Oncol. 2016; doi:10.1200/ JCO.2016.67.1529.

69. Weterman MA, Ajubi N, van Dinter IM, Degen WG, van Muijen GN, Ruitter DJ, et al. Nmb, a novel gene, is expressed in low-metastatic human melanoma cell lines and xenografts. Int J Cancer. 1995;60(1):73-81.

70. Tse KF, Jeffers M, Pollack VA, McCabe DA, Shadish ML, Khramtsov NV, et al. CR011, a fully human monoclonal antibody-auristatin E conjugate, for the treatment of melanoma. Clin Cancer Res. 2006;12(4):1373-82.

71. Doronina SO, Toki BE, Torgov MY, Mendelsohn BA, Cerveny CG, Chace DF, et al. Development of potent monoclonal antibody auristatin conjugates for cancer therapy. Nat Biotechnol. 2003;21(7):778-84.

72. Doronina SO, Bovee TD, Meyer DW, Miyamoto JB, Anderson ME, MorrisTilden CA, et al. Novel peptide linkers for highly potent antibodyauristatin conjugate. Bioconjug Chem. 2008;19(10):1960-3.

73. McDonagh CF, Turcott E, Westendorf L, Webster JB, Alley SC, Kim K, et al. Engineered antibody-drug conjugates with defined sites and stoichiometries of drug attachment. Protein Eng Des Sel. 2006;19(7):299-307.

74. Pollack VA, Alvarez E, Tse KF, Torgov MY, Xie S, Shenoy SG, et al. Treatment parameters modulating regression of human melanoma xenografts by an antibody-drug conjugate (CR011-vCMMAE) targeting GPNMB. Cancer Chemother Pharmacol. 2007;60(3):423-35.

75. Ott PA, Hamid O, Pavlick AC, Kluger H, Kim KB, Boasberg PD, et al. Phase I/II study of the antibody-drug conjugate glembatumumab vedotin in patients with advanced melanoma. J Clin Oncol. 2014;32(32):3659-66.

76. Thompson JF, Hersey P, Wachter E. Chemoablation of metastatic melanoma using intralesional rose bengal. Melanoma Res. 2008;18:405-11.

77. Delprat GD, Epstein NN, Kerr WJ. A new liver function test. The elimination of rose bengal when injected into the circulation of human subjects. Arch Intern Med. 1924;34:533-41.

78. Marsh RJ, Fraunfelder FT, McGill Jl. Herpetic corneal epithelial disease. Arch Ophthalmol. 1976;94:1899-902.

79. Wachter E, Dees C, Harkins J, Fisher W, Scott T. Imaging photosensitizer distribution and pharmacology using multiphoton microscopy. In: Farkas DL, Leif RC, editors. Proceedings of SPIE, optical diagnostics of living cells V. Bellingham: SPIE; 2002. p. 112-8.

80. Toomey P, Kodumudi K, Weber A, Kuhn L, Moore E, Sarnaik AA, et al. Intralesional injection of rose bengal induces a systemic tumor-specific immune response in murine models of melanoma and breast cancer. PLOS ONE. 2013:8:e68561.

81. Sarnaik A, Crago G, Liu H, Kodumudi K, Weber A, McCardle T, et al. Assessment of immune and clinical efficacy after intralesional PV-10 in injected and uninjected metastatic melanoma lesions. J Clin Oncol. 2014;32(Suppl 5s):9028.

82. Thompson JF, Agarwala SS, Smithers BM, Ross MI, Scoggins CR, Coventry BJ, et al. Phase 2 Study of Intralesional PV-10 in Refractory Metastatic Melanoma. Ann Surg Oncol. 2015:22:2135-42.

83. Andtbacka RHI, Ross MI, Agarwala SS, Taylor MH, Vetto JT, Neves RI, et al. Preliminary results from phase $\|$ study of combination treatment with HF10, a replication-competent HSV-1 oncolytic virus, and ipilimumab in patients with stage IIIb, IIlc, or IV unresectable or metastatic melanoma. J Clin Oncol. 2016;34:9543.

84. Eroglu Z, Gibney GT, Weber JS, Kudchadkar RR, Khushalani NI, Markowitz J, et al. Phase I study of vemurafenib and heat shock protein 90 (HSP90) inhibitor XL888 in metastatic BRAF V600 mutant melanoma. J Clin Oncol. 2016:34:9544. 